\title{
Initial Speeds of CMEs Estimated by Using Solar Wind Observations Near 1 AU
}

\author{
S. Watari ${ }^{1}$, M. Vandas $^{2}$ and T. Watanabe 3 \\ ${ }^{1}$ National Institute of Information and Communications Technology, \\ 4-2-1 Nukuikita, Koganei, Tokyo 184-8795, Japan \\ email: watari@nict.go.jp \\ ${ }^{2}$ Astronomical Institute, Academy of Sciences, \\ Boční II 1401, 14131 Praha 4, Czech Republic \\ email: vandas@ig.cas.cz \\ ${ }^{3}$ Ibaraki University, 2-1-1 Bunkyo, Mito, Ibaraki 310-8512, Japan \\ email: watanabe@env.sci.ibaraki.ac.jp
}

\begin{abstract}
It is an important subject of space weather to forecast accurate arrival time of interplanetary coronal mass ejections (ICMEs) at the Earth. Determination of initial speeds of CMEs is an important factor for this. Here, we estimated the initial speeds of CMEs using solar wind observations near $1 \mathrm{AU}$ and compared these speeds with CME speeds measured by the SOHO coronagraph.
\end{abstract}

Keywords. Solar wind, Sun:coronal mass ejections (CMEs), solar-terrestrial relations.

\section{Introduction}

Intense geomagnetic storms are often initiated by arrival of interplanetary coronal mass ejections (ICMEs). Therefore it is an important subject for space weather to predict arrival time of the ICMEs accurately. For the accurate prediction, we need to get accurate initial speeds of ICMEs as an input. The coronagraph on board the SOHO spacecraft enables to measure the CME speeds based on observations (Yashiro et al. 2004). The problem is that the measured CME speeds are apparent ones. Zhao, Plunkett \& Liu (2002) tried to determine radial speeds of CMEs using the cone model. Dal Lago, Schwenn \& Gonzalez (2003) presented a relationship between CME radial speeds and expansion speeds based on the "limb CME" observations. Here, we estimated the initial speeds of CME using solar wind speeds near $1 \mathrm{AU}$ and compared them with the measured CME speeds. We used a simple model (Watari \& Detman 1998) based on the shock time arrival (STOA) model developed by Dryer \& Smart (1984) for this.

\section{Comparison between measured and estimated speeds of CMEs}

We assumed distance dependence of ICME speed to be

$$
\begin{gathered}
V=V_{o}+V_{b} \quad \text { for } \quad R=R_{s} \sim R_{1} \\
V=V_{o}\left(\frac{R_{1}}{R}\right)^{\alpha}+V_{b} \quad \text { for } \quad R \geqslant R_{1}
\end{gathered}
$$

where $\mathrm{V}$ is speed of ICME at distance $\mathrm{R}$ from the Sun. $R_{s}$ is the solar radius. $V_{o}$ is initial speed of ICME. $V_{b}$ is background solar wind speed. Deceleration of ICME starts at $R_{1}$. 

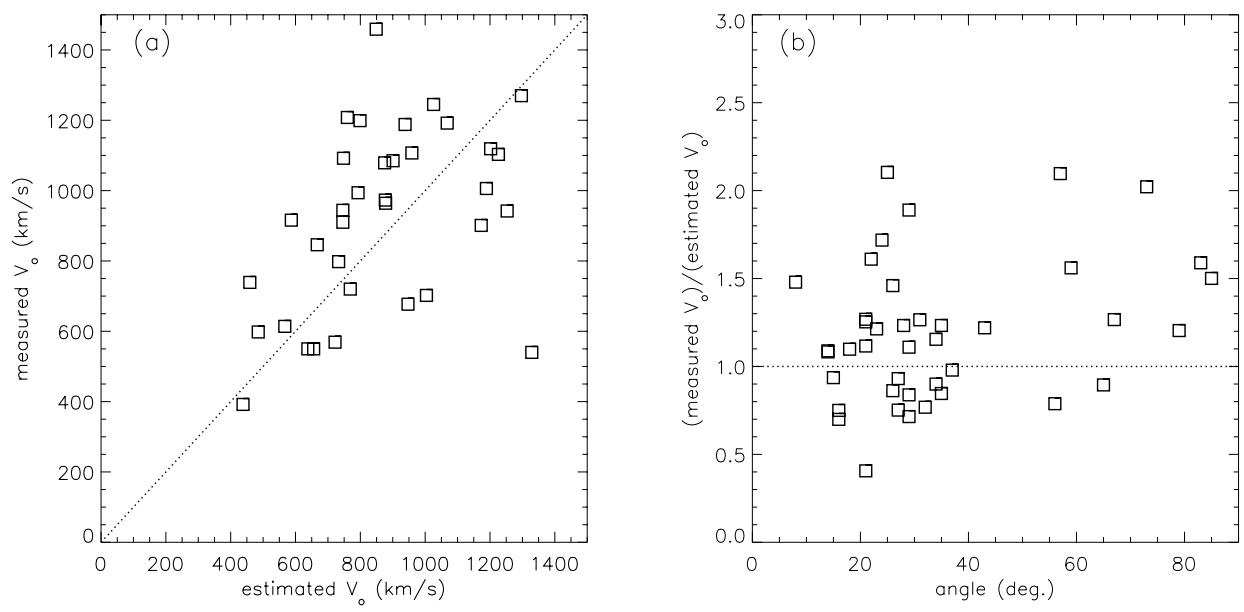

Figure 1. (a) A scatter plot of the measured and the estimated initial CME speeds. (b) A scatter plot of angles between Earth direction and main CME direction and ratios of the measured and estimated initial CME speeds.

We picked up 41 events which were clearly identified by the ACE observations between 2000 and 2003. For background solar wind speeds we choose solar wind speeds of quiet periods just before the shock arrival.

We assumed that $\alpha$ was 0.5 and $R_{1}$ was $0.15 \mathrm{AU}$ (based on the result by Watari \& Detman 1998) and estimated initial speeds of ICMEs using equation (2.2) and observed solar wind speeds near 1 AU. Figure 1a is a scatter plot of the estimated initial speeds of ICMEs and CME speeds measured by the SOHO observations. Figure 1b shows angles between Earth directions and main portions of CMEs and ratios of the measured and estimated speeds. The ratios tend to become larger as the angles increase. This shows the contribution of projection effect of CMEs in the measured CME speeds. The averaged value of the ratios for angles less than 45 degrees is approximately 1.1. This is consistent with the result of Dal Lago, Schwenn \& Gonzalez (2003). This suggests the contribution of the expansion effect in the measured CME speeds.

\section{Acknowledgements}

The ACE solar wind data was provided from the ACE Science Center. The measured CME speeds are referred from the catalog generated and maintained by NASA and The Catholic University of America in cooperation with the Naval Research Laboratory. SOHO is a project of international cooperation between NASA and ESA. MV was supported by projects S1003006 from AV ČR and ME501 from MŠMT ČR.

\section{References}

Dal Lago, A. Schwenn, R. \& Gonzalez, W. D. 2003, Adv. Space Res. 32, 2637

Dryer, M. \& Smart, D. F. 1984, Adv. Space Res. 4, 291

Yashiro, S., Gopalswamy, N., Michalek, G., Cyr, O. C. St., Plunkett, S. P., Rich, N. B., \& Howard, R. A. 2004, J. Geophys. Res. 109, A07105

Watari, S. \& Detman, T. 1998, Ann. Geophys. 16, 370

Zhao, X. P., Plunkett, S. P., \& Liu, W. 2002, J. Geophys. Res. 107, SSH13-1 